\title{
SIMPLE MULTIPLIERS ON BANACH MODULES
}

\author{
JANKO BRAČIČ \\ University of Ljubljana, IMFM, Jadranska 19, 1111 Ljubljana, Slovenia \\ e-mail:janko.bracic@guest.arnes.si
}

(Received 19 March, 2002; accepted 19 September, 2002)

\begin{abstract}
In this paper we introduce simple multipliers, a special subclass of multipliers on a Banach module. We show that, from a local spectral point of view, these multipliers behave like multipliers on a commutative Banach algebra. Our definition of simple multipliers relies on the notion of point multipliers. These multipliers were studied earlier. However our approach gives new insight into this topic and therefore could be of some interest by itself.
\end{abstract}

2000 Mathematics Subject Classification. Primary 46H25; Secondary 47B40.

1. Introduction. A mapping $T$ on a Banach algebra $\mathcal{A}$ is a multiplier if $x(T y)=$ (Tx)y for all $x, y \in \mathcal{A}$; see [13]. Denote by $\mathcal{M}(\mathcal{A})$ the set of all multipliers on $\mathcal{A}$. It can be proven, under some mild condition on $\mathcal{A}$, that $\mathcal{M}(\mathcal{A})$ is a closed commutative subalgebra in $B(\mathcal{A})$, the algebra of all bounded operators on $\mathcal{A}$. Moreover, in this case, we have $T(x y)=x(T y)=(T x) y, x, y \in \mathcal{A}$, for every multiplier on $\mathcal{A}$. The latter equality motivates us to call a bounded linear operator $T$, which maps from a left Banach $\mathcal{A}$-module $X$ into a left Banach $\mathcal{A}$-module $\mathcal{Y}$ ( $\mathcal{A}$ is not necessarily commutative), a multiplier if it satisfies $T(a \cdot x)=a \cdot T x$ for all $a \in \mathcal{A}, x \in X$. The set of all multipliers from $X$ into $y$ is denoted by $B_{\mathcal{A}}(X, y)$. (If $X=y$ we shall write $B_{\mathcal{A}}(X)$ instead of $B_{\mathcal{A}}(X, X)$ and in the case of right modules we will use the notation $B(X, Y)_{\mathcal{A}}$, resp. $\left.B(X)_{\mathcal{A}}\right)$.

The goal of the present paper is to discuss the decomposability of multipliers between Banach modules. Since, for a general multiplier, we cannot say very much for instance, if $\mathcal{A}=\mathbb{C}$, then $B_{\mathcal{A}}(\mathcal{X})=B(X)$ - it is quite clear that we have to confine ourselves to a special subclass of multipliers. In Section 4 we introduce simple multipliers on a Banach left module, which seem to be the suitable environment for our questions. Section 5 is devoted to the local spectral theory of simple multipliers. For instance, we extend to the class of simple multipliers on a left Banach module some results that are proven in [8], [16] and [23] only for algebras. Our definition of simple multipliers relies on the notion of point multipliers, which are studied in Sections 2 and 3. We believe that the content of these two sections are of some interest by itself.

It is assumed that the reader is familiar with the concept of Banach modules. We refer to $[\mathbf{1}, \mathbf{1 8}, \mathbf{2 4}]$, for details.

2. Point multipliers. Let $\mathcal{A}$ be a Banach algebra. Denote by $\Sigma(\mathcal{A})$ the set of all characters on $\mathcal{A}$, i.e. non-trivial multiplicative linear functionals, and let $\Sigma_{0}(\mathcal{A})$ denote the union $\Sigma(\mathcal{A}) \cup\{0\}$. The Banach space $\mathbb{C}$ is a left Banach $\mathcal{A}$-module if the 
multiplication is defined by $a \cdot z=\varphi(a) z$ for all $a \in \mathcal{A}$ and all $z \in \mathbb{C}$, where $\varphi \in \Sigma_{0}(\mathcal{A})$. This module will be denoted by $\mathbb{C}_{\varphi}$.

Definition 2.1. Let $X$ be a Banach left $\mathcal{A}$-module and let $\varphi \in \Sigma_{0}(\mathcal{A})$. A linear functional $\xi \in X^{*}$ is a point multiplier at $\varphi$ on $X$ if it is a multiplier from $X$ into $\mathbb{C}_{\varphi}$, i.e.

$$
\langle\xi, a \cdot x\rangle=\varphi(a)\langle\xi, x\rangle \quad \text { for all } a \in \mathcal{A}, x \in X .
$$

Point multipliers have been used earlier (under different names) by some other authors. See, for instance, Proposition 4 in $[1, \S 43]$, for a connection with amenability, and [12] for a connection with representation of Banach modules. See also [2].

If a Banach algebra $\mathcal{A}$ is without a unit, let $\mathcal{A}_{1}:=\mathcal{A} \oplus \mathbb{C}$ be its standard unitization. It is easily seen that each left Banach $\mathcal{A}$-module $X$ is also a left Banach $\mathcal{A}_{1}$-module under the multiplication $(a \oplus \lambda) \cdot x=a \cdot x+\lambda x$. A functional $\xi \in X^{*}$ is a point multiplier at $\varphi \in \Sigma_{0}(\mathcal{A})$ if and only if $\xi$ is a point multiplier at $\tilde{\varphi} \in \Sigma\left(\mathcal{A}_{1}\right)$, where $\tilde{\varphi}$ is given by $\widetilde{\varphi}(a \oplus \lambda):=\varphi(a)+\lambda$. Hence, if we are interested in the point multipliers, then there is no loss of generality if we assume that $\mathcal{A}$ is a unital Banach algebra.

Now we are going to give some examples of point multipliers. For a character $\varphi$ we shall denote by $M_{\varphi}$ the kernel $\operatorname{ker} \varphi$, i.e. the maximal ideal in $\mathcal{A}$ that corresponds to $\varphi$.

ExAmple 2.2. Let $\mathcal{A}$ be a unital Banach algebra and consider $\mathcal{A}$ as a left $\mathcal{A}$ module via the left regular representation. Then it is easily seen that there do not exist non-trivial point multipliers at 0 . On the other hand, there exist point multipliers at $\varphi \in \Sigma(\mathcal{A})$. Namely, a linear functional $\xi \in \mathcal{A}^{*}$ is a point multiplier at $\varphi$ if and only if there is $\alpha \in \mathbb{C}$ such that $\xi=\alpha \varphi$.

Let $\varphi \in \Sigma(\mathcal{A})$ and consider $M_{\varphi}$ as a left $\mathcal{A}$-module. If $\psi \in \Sigma(\mathcal{A})$ and $\psi \neq \varphi$, then $\left\{\left.\alpha \psi\right|_{M_{\varphi}} ; \alpha \in \mathbb{C}\right\}$, where $\left.\psi\right|_{M_{\varphi}}$ is the restriction of $\psi$ to $M_{\varphi}$, is the set of all point multipliers on $M_{\varphi}$ at $\psi$. On the other hand, point multipliers on $M_{\varphi}$ at $\varphi$ are exactly the functionals in ${\overline{M_{\varphi}^{2}}}^{\perp}\left(\subseteq M_{\varphi}^{*}\right)$.

EXAMPLE 2.3. Let $\mathcal{A}$ be a commutative Banach algebra and let $\mathcal{I}$ be a dense ideal in $\mathcal{A}$. Assume that there is a norm $\|\cdot\|^{\prime}$ on $\mathcal{I}$ such that $\mathcal{I}$ is a Banach algebra with respect to this norm and we have

$$
\|a x\|^{\prime} \leq\|a\|\|x\|^{\prime} \quad \text { for all } a \in \mathcal{A}, x \in \mathcal{I},
$$

where $\|\cdot\|$ denotes the norm on $\mathcal{A}$. Then $\mathcal{I}$ is a Banach left $\mathcal{A}$-module. The restriction $\left.\varphi\right|_{\mathcal{I}}$ of a character $\varphi \in \Sigma(\mathcal{A})$ to the ideal $\mathcal{I}$ is a character on $\mathcal{I}$. The mapping $\left.\varphi \mapsto \varphi\right|_{\mathcal{I}}$ is a bijection from $\Sigma(\mathcal{A})$ onto $\Sigma(\mathcal{I})$. It follows that $\left.\varphi\right|_{\mathcal{I}}$ is a point multiplier at $\varphi \in \Sigma(\mathcal{A})$. Let now $\xi$ be an arbitrary non-trivial point multiplier at $\varphi$. Since $\mathcal{I}$ is dense in $\mathcal{A}$ there is $e \in \mathcal{I}$ such that $\varphi(e)=1$. Hence

$$
\langle\xi, x\rangle=\langle\xi, e x\rangle=\langle\xi, x e\rangle=\varphi(x)\langle\xi, e\rangle \text { for all } x \in \mathcal{I}
$$

and therefore $\xi=\left.\langle\xi, e\rangle \varphi\right|_{\mathcal{I}}$.

EXAMPLE 2.4. Let $G$ be a locally compact abelian group. It is well known that each Banach space $L^{p}(G)$, where $1<p<\infty$, is a left Banach module over the group algebra $L^{1}(G)$ (the algebra $L^{1}(G)$ acts on $L^{p}(G)$ through the convolution). Note that 
the spectrum $\Sigma\left(L^{1}(G)\right)$ of the group algebra can be identified with the dual group $\widehat{G}$ (see $[24, \S 3.6])$.

Since $L^{1}(G) * L^{p}(G)=L^{p}(G)$ (see Example 16.6 in [6]) there do not exist non-trivial point multipliers at 0 .

If the group $G$ is compact, then $L^{p}(G)$ is a dense ideal in $L^{1}(G)$ and we have

$$
\|f * g\|_{p} \leq\|f\|_{1}\|g\|_{p} \quad \text { for all } f \in L^{1}(G), g \in L^{p}(G) .
$$

Hence, by the preceding example, point multipliers at $\varphi \in \widehat{G}$ form an one-dimensional subspace in $\left(L^{p}(G)\right)^{*}$.

Assume now that the group $G$ is not compact. Let $\mathcal{C}_{c}(G)$ be the algebra of all continuous functions on $G$ which have compact support. Suppose that $\xi \in\left(L^{q}(G)\right)^{*}$ is a point multiplier at $\varphi \in \widehat{G}$. Then

$$
\langle\xi, f * g\rangle=\varphi(f)\langle\xi, g\rangle \text { for all } f, g \in \mathcal{C}_{c}(G) .
$$

Since $\mathcal{C}_{c}(G)$ is dense in $L^{p}(G)$ there exists $e \in \mathcal{C}_{c}(G)$ such that $\varphi(e)=1$. It follows from (1) that $\left.\xi\right|_{\mathcal{e}_{c}(G)}=\langle\xi, e\rangle \varphi \mid \mathcal{e}_{c}(G)$. If the number $\langle\xi, e\rangle$ were non-zero, then $\eta:=\langle\xi, e\rangle^{-1} \xi$ would be a continuous linear functional on $L^{p}(G)$ such that $\left.\eta\right|_{\mathcal{E}_{c}(G)}=\varphi \mid \mathcal{e}_{c}(G)$. But this is a contradiction because $\left.\varphi\right|_{\mathcal{C}_{c}(G)}$ cannot be continuously extended to $L^{p}(G)$. Thus there do not exist non-trivial point multipliers at $\varphi \in \widehat{G}$.

Denote by $X_{\varphi}, \varphi \in \Sigma(\mathcal{A})$, the closure of the set

$$
M_{\varphi} \cdot X:=\left\{\sum_{k=1}^{n} a_{k} \cdot x_{k} ; a_{k} \in M_{\varphi}, x_{k} \in X, k=1, \ldots, n\right\} .
$$

It is clear that $X_{\varphi}$ is a submodule in $X$. Moreover, if $Z \subseteq X$ is a subspace such that $x_{\varphi} \subseteq Z$ for some $\varphi \in \Sigma(\mathcal{A})$, then $Z$ is a submodule as well.

Definition 2.5. Let $\mathcal{A}$ be a Banach algebra and let $\mathcal{X}$ be a Banach left $\mathcal{A}$-module. The Gelfand radical of $X$ is the submodule

$$
\operatorname{Rad}_{\mathcal{A}}(\mathcal{X}):=\cap\left\{X_{\varphi} ; \varphi \in \Sigma_{0}(\mathcal{A})\right\}
$$

in $X$.

We shall say that $\mathcal{X}$ is hyper-semisimple if its Gelfand radical is trivial. Of course, the counterpart of Gelfand radical in the theory of commutative Banach algebras is the ordinary Gelfand radical. Note that there exists a pure algebraic theory about radicals in modules over rings. (See [26] and references cited there.)

EXAMPLE 2.6. Let $G$ be a locally compact abelian group and $1<p<\infty$. It follows from Example 2.4 that $L^{p}(G)$ is a hyper-semisimple left Banach $L^{1}(G)$-module if $G$ is compact. Indeed, if a function $f$ is in the Gelfand radical of $L^{p}(G)$, then we have $\langle\varphi, f\rangle=$ $\int_{G} f(x) \varphi(x) d x=0$ for all $\varphi \in G$ (note that $\varphi$ is regarded as an element in $L^{q}(G)=$ $\left(L^{p}(G)\right)^{*}$, where $\left.1 / p+1 / q=1\right)$. Define $g(x):=f(-x), x \in G$. Then $g \in L^{p}(G) \subseteq L^{1}(G)$ and hence

$$
0=\langle\varphi, f\rangle=\int_{G} f(x) \varphi(x) d x=\int_{G} g(x) \varphi(-x) d x=\widehat{g}(\varphi),
$$


where $\widehat{g}$ is the Fourier transform of $g$. Since the group algebra is semisimple the function $g$ is trivial and so is $f$.

If $G$ is not compact, then $\operatorname{Rad}_{L^{1}(G)}\left(L^{p}(G)\right)=L^{p}(G)$, by Example 2.4.

Let $\mathcal{X}$ be a Banach left $\mathcal{A}$-module. We shall say that a non-zero vector $x \in \mathcal{X}$ is a characteristic vector if there exists $\varphi \in \Sigma_{0}(\mathcal{A})$ satisfying $a \cdot x=\varphi(a) x$ for all $a \in \mathcal{A}$. Let

$$
C h_{\varphi}(X):=\{x \in X ; a \cdot x=\varphi(a) x \text { for all } a \in \mathcal{A}\} .
$$

It is easily seen that this is a submodule in $X$.

The topological dual $X^{*}$ of a left Banach $\mathcal{A}$-module $X$ is a right Banach $\mathcal{A}$-module for the multiplication $\langle\xi \cdot a, x\rangle=\langle\xi, a \cdot x\rangle, \xi \in \mathcal{X}^{*}, a \in \mathcal{A}, x \in \mathcal{X}$. Each submodule $C h_{\varphi}\left(X^{*}\right)$ is weak-* closed. Let $C h_{\mathcal{A}}(X)$ be the closure of the linear span of the union $\cup\left\{C h_{\varphi}(X) ; \varphi \in \Sigma_{0}(\mathcal{A})\right\}$ and let $C h_{\mathcal{A}}^{*}\left(X^{*}\right)$ be the weak-* closure of the linear span of the union $\cup\left\{C h_{\varphi}\left(X^{*}\right) ; \varphi \in \Sigma_{0}(\mathcal{A})\right\}$. Then $C h_{\mathcal{A}}(X)$ is a submodule in $X$ and $C h_{\mathcal{A}}^{*}\left(X^{*}\right)$ is a weak-* closed submodule in $X^{*}$.

Recall that $y^{\perp}:=\left\{\eta \in X^{*} ;\langle\eta, y\rangle=0\right.$ for all $\left.y \in y\right\}$ is a weak-* closed subspace in $X^{*}$ for each linear manifold $y$ in a Banach space $X$. Moreover, if $X$ is a left Banach $\mathcal{A}$-module and $y$ is a submodule, then $y^{\perp}$ is a submodule in the dual $\mathcal{A}$-module $X^{*}$. Similarly, if $\mathcal{W}$ is a linear manifold in $\mathcal{X}^{*}$, then $\mathcal{W}_{\perp}:=\{x \in \mathcal{X} ;\langle\eta, x\rangle=0$ for all $\eta \in \mathcal{W}\}$ is a closed subspace in $X$, and $\left(\mathcal{W}_{\perp}\right)^{\perp}$ is the weak-* closure of $\mathcal{W}$ in $X^{*}$, (cf. [4, p. 130], if $y$ is a closed subspace in $X$, then $\left(y^{\perp}\right)_{\perp}=y$ by the Hahn-Banach theorem). Again, if $\mathcal{X}$ is a left Banach $\mathcal{A}$-module and $\mathcal{W}$ is an $\mathcal{A}$-submodule in the dual $\mathcal{A}$-module $X^{*}$, then $\mathcal{W}_{\perp}$ is an $\mathcal{A}$-submodule in $X$.

Proposition 2.7. Let $\mathcal{A}$ be a unital Banach algebra and let $\mathcal{X}$ be a left Banach $\mathcal{A}$-module. Then

$$
C h_{\varphi}\left(X^{*}\right)=B_{\mathcal{A}}\left(X, \mathbb{C}_{\varphi}\right)=X_{\varphi}^{\perp}
$$

for each $\varphi \in \Sigma(\mathcal{A})$, where $X^{*}$ is considered as a dual Banach $\mathcal{A}$-module. Moreover,

$$
\operatorname{Ch}_{\mathcal{A}}^{*}\left(X^{*}\right)=\operatorname{Rad}_{\mathcal{A}}(X)^{\perp} .
$$

Proof. The equations in (2) can be easily checked. Since $\operatorname{Rad}_{\mathcal{A}}(X)$ is included in $X_{\varphi}$, for every $\varphi \in \Sigma(\mathcal{A})$, we have $X_{\varphi}^{\perp} \subseteq \operatorname{Rad}_{\mathcal{A}}(\mathcal{X})^{\perp}$. By $(2), C h_{\mathcal{A}}^{*}\left(X^{*}\right)$ is the weak-* closure of the linear span of the union $\cup\left\{X_{\varphi}^{\perp} ; \varphi \in \Sigma(\mathcal{A})\right\}$ and therefore, since $\operatorname{Rad}_{\mathcal{A}}(X)^{\perp}$ is weak-* closed, it is contained in $\operatorname{Rad}_{\mathcal{A}}(\mathcal{X})^{\perp}$.

Assume now that $\xi \in X^{*}$ is not in $C h_{\mathcal{A}}^{*}\left(X^{*}\right)$. If $k e r \xi=\{\xi\}_{\perp}$ contained $C h_{\mathcal{A}}^{*}\left(X^{*}\right)_{\perp}$, then $\left(\{\xi\}_{\perp}\right)^{\perp}$ would be contained in $\left(C h_{\mathcal{A}}^{*}\left(\mathcal{X}^{*}\right)_{\perp}\right)^{\perp}$. However, this is not the case. Thus, there is $x \in C h_{\mathcal{A}}^{*}\left(X^{*}\right)_{\perp}$ such that $\langle\xi, x\rangle \neq 0$. Since $X_{\varphi}^{\perp} \subseteq C h_{\mathcal{A}}^{*}\left(X^{*}\right)$ for all $\varphi \in \Sigma(\mathcal{A})$, we have $x \in C h_{\mathcal{A}}^{*}\left(\mathcal{X}^{*}\right)_{\perp} \subseteq X_{\varphi}$ for all $\varphi \in \Sigma(\mathcal{A})$. Hence, $x$ is in $\operatorname{Rad}_{\mathcal{A}}(X)$ and it follows that $\xi \notin \operatorname{Rad}_{\mathcal{A}}(\mathcal{X})^{\perp}$.

3. Submodules. Let $\mathcal{A}$ be a Banach algebra and let $\mathcal{X}$ be a left Banach $\mathcal{A}$-module. If $\xi \in X^{*}$ is a non-trivial point multiplier, then ker $\xi$ is a submodule in $X$ with codimension 1. On the other hand, it is not hard to see that for each closed submodule $\mathcal{P}$ of co-dimension 1 in $X$ there exists a point multiplier $\xi$ on $X$ such that $\mathcal{P}=k e r \xi$. We will denote by $\Delta_{\mathcal{A}}(\mathcal{X})$ the set of all closed submodules in $\mathcal{X}$ which have co-dimension 1 . 
The set of all those submodules in $\Delta_{\mathcal{A}}(\mathcal{X})$ that correspond to $\varphi \in \Sigma(\mathcal{A})$, i.e., the set of null-spaces of point multipliers in $C h_{\varphi}\left(X^{*}\right) \backslash\{0\}$, will be denoted by $\Delta_{\varphi}(X)$.

The hull-kernel topology is very important notion in the theory of Banach algebras. It can be defined on each non-empty subset of the set of prime ideals of an algebra. See $[\mathbf{2 4}, \S 7.1]$. If $\mathcal{A}$ is a unital Banach algebra, then there is a bijective correspondence between points in $\Sigma(\mathcal{A})$ and closed prime ideals with co-dimension 1 . Thus, a point in $\Sigma(\mathcal{A})$ can be considered as a non-trivial multiplicative linear functional as well as a prime ideal of co-dimension 1 . Recall that the hull of a subset $\mathcal{U} \subseteq \mathcal{A}$ is the set $h_{\mathcal{A}}(\mathcal{U})=\left\{\varphi \in \Sigma(\mathcal{A}) ; \mathcal{U} \subseteq M_{\varphi}\right\}$ and that the kernel of a subset $S \subseteq \Sigma(\mathcal{A})$ is the set $k_{\mathcal{A}}(S)=\cap\left\{M_{\varphi} ; \varphi \in S\right\}$. The family $\left\{\Sigma(\mathcal{A}) \backslash h_{\mathcal{A}}\left(k_{\mathcal{A}}(S)\right) ; S \subseteq \Sigma(\mathcal{A})\right\}$ is the hull-kernel topology on $\Sigma(\mathcal{A})$, (cf. [24]).

Let $\mathcal{A}$ be a unital Banach algebra and let $X$ be a left Banach $\mathcal{A}$-module. The annihilator of a non-empty subset $\mathcal{M} \subseteq \mathcal{X}$ is $\operatorname{ann}_{\mathcal{A}}(\mathcal{M}):=\{a \in \mathcal{A} ; a \cdot x=0$ for all $x \in$ $\mathcal{M}\}$. Following $[\mathbf{1 9}, \mathbf{2 0}, \mathbf{2 1}]$ we shall also say that the annihilator of a quotient $\mathcal{A}$-module $x / y$, where $y$ is a submodule in $X$, is the quotient of a submodule $y$ and we will denote it by $(y: X)$. Thus, $(y: X):=\{a \in \mathcal{A} ; a \cdot X \subseteq Y\}$. It is easily seen that $(y: X)$ is a closed two-sided ideal in $\mathcal{A}$ and it always contains the annihilator $a n_{\mathcal{A}}(X)$. Of course, the quotient of the trivial submodule 0 is $\operatorname{ann}_{\mathcal{A}}(X)$ and $(X: X)=\mathcal{A}$. If $y$ and $Z$ are submodules in $X$ such that $y \subseteq Z$, then $(y: X) \subseteq(Z: X)$. The quotient of $\mathcal{P} \in \Delta_{\varphi}(X)$ is the maximal ideal $M_{\varphi}$.

Definition 3.1. Let $\mathcal{A}$ be a unital Banach algebra and let $\mathcal{X}$ be a left Banach $\mathcal{A}$-module. The kernel of a non-empty subset $S \subseteq \Delta_{\mathcal{A}}(\mathcal{X})$ is $k_{\mathcal{X}}(S):=\cap_{\mathcal{P} \in S} \mathcal{P}$ and the kernel of the empty set is $X$. The hull of a submodule $y$ in $X$ is

$$
h_{X}(y):=\left\{\mathcal{P} \in \Delta_{\mathcal{A}}(X) ;(y: X) \subseteq(\mathcal{P}: X)\right\}
$$

The idea of how the hulls are defined comes from [20] (see also [19] and [21] and references cited therein). However, we shall make our approach more similar to that in the theory of Banach algebras.

The kernel of any subset $S \subseteq \Delta_{\mathcal{A}}(X)$ is a submodule in $X$ and the Gelfand radical of $X$ is included in it. In the next proposition some properties of the hulls and kernels are listed. We will use the following notation. If $\left\{\mathcal{I}_{i}\right\}_{i \in I}$ is a family of left ideals in $\mathcal{A}$, then $\sum_{i \in I} \mathcal{I}_{i} \cdot X$ is the linear span of the set $\left\{a \cdot x ; a \in \mathcal{I}_{i}\right.$ for some $i$ and $\left.x \in X\right\}$. Of course, the closure $\overline{\sum_{i \in I} \mathcal{I}_{i} \cdot X}$ is a submodule in $X$.

Proposition 3.2. Let $\mathcal{A}$ be a unital Banach algebra and let $X$ be a Banach left $\mathcal{A}$-module.

(i) $h_{X}(0)=\Delta_{\mathcal{A}}(X), h_{X}(X)=\emptyset, k_{X}(\emptyset)=X$ and $k_{X}\left(\Delta_{\mathcal{A}}(X)\right)=\operatorname{Rad}_{\mathcal{A}}(X)$.

(ii) If $S_{1} \subseteq S_{2} \subseteq \Delta_{\mathcal{A}}(\mathcal{X})$, then $k_{X}\left(S_{1}\right) \supseteq k_{X}\left(S_{2}\right)$, and $h_{X}(Z) \supseteq h_{X}(y)$ if $y$ and $z$ are submodules in $X$ such that $\mathcal{Z} \subseteq \mathcal{Y}$.

(iii) For any subset $S$ of $\Delta_{\mathcal{A}}(X)$ we have $S \subseteq h_{x}\left(k_{x}(S)\right)$.

(iv) If $y$ is a submodule in $X$, then $h_{x}(y)=h_{x}\left(k_{x}\left(h_{x}(y)\right)\right)$.

(v) Let $\left\{y_{i}\right\}_{i \in I}$ be a family of submodules of $X$. Then

$$
\cap_{i \in I} h_{X}\left(y_{i}\right)=h_{X}\left(\overline{\sum_{i \in I}\left(y_{i}: X\right) \cdot X}\right) .
$$

(vi) If $y$ and $z$ are submodules in $x$, then $h_{x}(y) \cup h_{x}(z)=h_{x}(y \cap z)$. 
Proof. This proof relies on [20]. Short computations show that (i), (ii) and (iii) are valid.

(iv) The inclusion $h_{x}(y) \subseteq h_{x}\left(k_{x}\left(h_{x}(y)\right)\right)$ follows from (iii). Assume that $\mathcal{P}_{0} \in$ $\Delta_{\mathcal{A}}(X)$ is such that $\mathcal{P}_{0} \notin h_{X}(Y)$. Then there exists $a \in(y: X)$ such that $a \notin\left(\mathcal{P}_{0}: X\right)$. Since $a \in(y: X) \subseteq(\mathcal{P}: X)$ for all $\mathcal{P} \in h_{X}(y)$ we have $a \cdot X \subseteq k_{X}\left(h_{X}(y)\right)$. Thus, $a$ is in the quotient of $k_{x}\left(h_{x}(y)\right)$ and therefore $\left(k_{x}\left(h_{x}(y)\right): X\right) \not \subset\left(\mathcal{P}_{0}: X\right)$.

(v) If $\mathcal{P}$ is in $\cap_{i \in I} h_{x}\left(y_{i}\right)$, then the quotient of each $y_{i}$ is included in the quotient of $\mathcal{P}$. It follows that $\sum_{i \in I}\left(y_{i}: X\right) \cdot X$ is included in $\mathcal{P}$ and since $\mathcal{P}$ is closed it contains also the closure of $\sum_{i \in I}\left(y_{i}: X\right) \cdot X$. Thus $\mathcal{P} \in h_{X}\left(\overline{\sum_{i \in I}\left(y_{i}: X\right) \cdot X}\right)$.

On the other hand, if $\mathcal{P}$ is in $h x\left(\overline{\sum_{i \in I}\left(y_{i}: X\right) \cdot X}\right)$, then it follows from

$$
\left(y_{i}: x\right) \subseteq\left(\overline{\left(y_{i}: X\right) \cdot x}: x\right) \subseteq\left(\overline{\sum_{i \in I}\left(y_{i}: x\right) \cdot x}: x\right) \subseteq(\mathcal{P}: X),
$$

that $\mathcal{P} \in \cap_{i \in I} h_{x}\left(y_{i}\right)$.

(vi) If $\mathcal{P} \in h_{X}(Y) \cup h_{X}(Z)$, then $(y: X) \subseteq(\mathcal{P}: X)$ or $(\mathcal{Z}: X) \subseteq(\mathcal{P}: X)$. Hence in any case $(y \cap z: X) \subseteq(\mathcal{P}: X)$ because the intersection $y \cap z$ is included in $y$ and in $z$.

To see that the opposite inclusion is valid we will show first that the product $(y: X)(z: X):=\left\{\sum_{k=1}^{n} a_{k} b_{k} ; a_{k} \in(y: X), b_{k} \in(Z: X)\right\}$ is contained in $(y \cap z: X)$. Indeed, let $x$ be an arbitrary vector from $X$. Then we have $\left(\sum_{k=1}^{n} a_{k} b_{k}\right) \cdot x \in y \cap z$ because $\sum_{k=1}^{n} a_{k} \cdot\left(b_{k} \cdot x\right) \in \mathcal{y}$, by definition of $(y: X)$, and $\sum_{k=1}^{n} a_{k} \cdot\left(b_{k} \cdot x\right) \in \mathcal{Z}$, by definition of $(Z: X)$ and since $Z$ is a submodule.

Let $\mathcal{P}$ be in $h_{x}(y \cap Z)$ and let $\varphi \in \Sigma(\mathcal{A})$ be the corresponding character on $\mathcal{A}$. Since $M_{\varphi}$ is a prime ideal in $\mathcal{A}$ it follows from

$$
(y: X)(Z: X) \subseteq(y \cap Z: X) \subseteq(\mathcal{P}: X)=M_{\varphi}
$$

that $(y: X) \subseteq(\mathcal{P}: X)$ or $(Z: X) \subseteq(\mathcal{P}: X)$.

Corollary 3.3. Let $\mathcal{A}$ be a unital Banach algebra and let $X$ be a Banach left $\mathcal{A}$-module. The correspondence $S \rightarrow h_{x}\left(k_{x}(S)\right), S \subseteq \Delta_{\mathcal{A}}(X)$, is a closure operation.

For a submodule $y$ in $X$, let $\omega(y)$ denote the complement of $h_{X}(y)$ in $\Delta_{\mathcal{A}}(X)$. It follows, by Corollary 3.3 , that the family $\{\omega(y) ; y$ is a submodule in $X\}$ is a topology, called the hull-kernel topology on $\Delta_{\mathcal{A}}(\mathcal{X})$. Note that this topology is sometimes called Zariski topology (cf. [19]). If a subset $U \subseteq \Delta_{\mathcal{A}}(X)$ is open in the hull-kernel topology, then $U=\omega\left(k_{x}\left(U^{c}\right)\right)$. It follows from Proposition 3.2 that the intersection of sets $\omega\left(y_{1}\right), \ldots, \omega\left(y_{n}\right)$ is $\omega\left(y_{1} \cap \ldots \cap y_{n}\right)$ and the union of a family $\left\{\omega\left(y_{i}\right)\right\}_{i \in I}$ is $\omega\left(\overline{\sum_{i \in I}\left(y_{i}: X\right) \cdot X}\right)$.

Singletons in $\Delta_{\mathcal{A}}(X)$ are not necessarily closed in the hull-kernel topology. If $\mathcal{P}_{0}$ is in $\Delta_{\varphi}(\mathcal{X})$ for some $\varphi \in \Sigma(\mathcal{A})$, then its closure is exactly $\Delta_{\varphi}(\mathcal{X})$. It follows that $\Delta_{\varphi}(\mathcal{X}) \subseteq S$ if $S \subseteq \Delta_{\mathcal{A}}(X)$ is hull-kernel closed and contains at least one point from $\Delta_{\varphi}(X)$. The same is true for hull-kernel open subsets in $\Delta_{\mathcal{A}}(X)$.

For $\mathcal{P}$ in $\Delta_{\mathcal{A}}(\mathcal{X})$ let $v_{\mathcal{A}}(\mathcal{P})$ be the corresponding character in $\Sigma(\mathcal{A})$. We shall say that $\nu_{\mathcal{A}}: \Delta_{\mathcal{A}}(X) \rightarrow \Sigma(\mathcal{A})$ is the natural map.

Proposition 3.4. Let $\mathcal{A}$ be a unital Banach algebra and let $X$ be a left Banach $\mathcal{A}$-module.

(i) For each two-sided ideal $\mathcal{I}$ in $\mathcal{A}$ we have

$$
h_{\mathcal{A}}(\mathcal{I}) \cap v_{\mathcal{A}}\left(\Delta_{\mathcal{A}}(X)\right)=v_{\mathcal{A}}\left(\left\{\mathcal{P} \in \Delta_{\mathcal{A}}(X) ; \mathcal{I} \subseteq(\mathcal{P}: X)\right\}\right) .
$$

(ii) If $S \subseteq \Delta_{\mathcal{A}}(X)$, then $k_{\mathcal{A}}\left(v_{\mathcal{A}}(S)\right)=\left(k_{X}(S): X\right)$. 
Proof. (i) Obvious.

(ii) For $\mathcal{P} \in S$ we have $(\mathcal{P}: \mathcal{X})=M_{\varphi}$ for some $\varphi \in v_{\mathcal{A}}(S)$, which implies $k_{\mathcal{A}}\left(v_{\mathcal{A}}(S)\right) \subseteq(\mathcal{P}: X)$. Thus, $k_{\mathcal{A}}\left(v_{\mathcal{A}}(S)\right) \cdot \mathcal{X} \subseteq \mathcal{P}$ for all $\mathcal{P} \in S$ and therefore $k_{\mathcal{A}}\left(v_{\mathcal{A}}(S)\right)$. $X \subseteq \cap_{\mathcal{P} \in S} \mathcal{P}=k_{X}(S)$. The opposite inclusion follows from the fact that $\left(k_{X}(S): X\right) \subseteq$ $(\mathcal{P}: X)$ for all $\mathcal{P} \in S$.

Proposition 3.5. If $\Delta_{\mathcal{A}}(\mathcal{X})$ and $\Sigma(\mathcal{A})$ are endowed with the hull-kernel topologies, then the natural map is continuous. If $S \subseteq \Delta_{\mathcal{A}}(X)$ is hull-kernel closed (open), then $v_{\mathcal{A}}(S)$ is closed (open) in the relative hull-kernel topology on $\nu_{\mathcal{A}}\left(\Delta_{\mathcal{A}}(X)\right)$. In particular, if $\nu_{\mathcal{A}}$ is surjective, then it maps hull-kernel closed (open) sets into hull-kernel closed (open) sets.

Proof. Let $F \subseteq \Sigma(\mathcal{A})$ be hull-kernel closed. Denote $\mathcal{F}=v_{\mathcal{A}}^{-1}(F)$. We have to show that $\mathcal{F}$ is hull-kernel closed. It is enough to see that $h_{x}\left(k_{X}(\mathcal{F})\right) \subseteq \mathcal{F}$ since the opposite inclusion follows from Proposition 3.2 (iii). Let $\mathcal{P} \in \Delta_{\varphi}(\mathcal{X})$ be in $h_{x}\left(k_{x}(\mathcal{F})\right)$. Since $v_{\mathcal{A}}(\mathcal{F}) \subseteq F$, we have

$$
k_{\mathcal{A}}(F) \subseteq k_{\mathcal{A}}\left(v_{\mathcal{A}}(\mathcal{F})\right)=\left(k_{X}(\mathcal{F}): X\right) \subseteq(\mathcal{P}: X)=M_{\varphi}
$$

Hence $\varphi \in h_{\mathcal{A}}\left(k_{\mathcal{A}}(F)\right)=F$ and therefore $\mathcal{P} \in v_{\mathcal{A}}^{-1}(F)=\mathcal{F}$.

If $S \subseteq \Delta_{\mathcal{A}}(\mathcal{X})$ is hull-kernel closed, then $v_{\mathcal{A}}(S)=h_{\mathcal{A}}\left(\left(k_{X}(S): \mathcal{X}\right)\right) \cap v_{\mathcal{A}}\left(\Delta_{\mathcal{A}}(X)\right)$, by (i) of the preceding proposition. Thus, $v_{\mathcal{A}}(S)$ is closed in the relative hull-kernel topology on $v_{\mathcal{A}}\left(\Delta_{\mathcal{A}}(\mathcal{X})\right)$. The rest of the proof is routine.

Proposition 3.6. Let $\mathcal{A}$ be a unital Banach algebra and let $\mathcal{X}$ be a hyper-semisimple left Banach $\mathcal{A}$-module. Then ann $n_{\mathcal{A}}(X)=k_{\mathcal{A}}\left(v_{\mathcal{A}}\left(\Delta_{\mathcal{A}}(X)\right)\right)$ and $v_{\mathcal{A}}$ is surjective if and only if $\operatorname{ann}_{\mathcal{A}}(X)=\operatorname{Rad}_{\mathcal{A}}(\mathcal{A})\left(=k_{\mathcal{A}}(\Sigma(\mathcal{A}))\right)$.

Proof. Since $X$ is hyper-semisimple we have $k_{X}\left(\Delta_{\mathcal{A}}(X)\right)=0$. Set $S=\Delta_{\mathcal{A}}(X)$ in Proposition 3.4 (ii). Then

$$
k_{\mathcal{A}}\left(v_{\mathcal{A}}\left(\Delta_{\mathcal{A}}(X)\right)\right)=\left(k_{X}\left(\Delta_{\mathcal{A}}(X): X\right)=(0: X)=\operatorname{ann}_{\mathcal{A}}(X)\right.
$$

If the natural map is surjective, then $\operatorname{ann}_{\mathcal{A}}(\mathcal{X})=k_{\mathcal{A}}(\Sigma(\mathcal{A}))=\operatorname{Rad}_{\mathcal{A}}(\mathcal{A})$. On the other hand, since $\Delta_{\mathcal{A}}(X)$ is hull-kernel closed its image $\nu_{\mathcal{A}}\left(\Delta_{\mathcal{A}}(X)\right)$ is hull-kernel closed in $\Sigma(\mathcal{A})$. Thus, if $a n n_{\mathcal{A}}(X)=\operatorname{Rad}_{\mathcal{A}}(\mathcal{A})$, then $v_{\mathcal{A}}\left(\Delta_{\mathcal{A}}(X)\right)=h_{\mathcal{A}}\left(k_{\mathcal{A}}\left(v_{\mathcal{A}}\left(\Delta_{\mathcal{A}}(X)\right)\right)\right)=$ $h_{\mathcal{A}}\left(k_{\mathcal{A}}(\Sigma(\mathcal{A}))\right)=\Sigma(\mathcal{A})$.

Let $\mathcal{A}$ be a unital Banach algebra and let $\mathcal{X}$ be a Banach left $\mathcal{A}$-module. For each $\mathcal{P} \in \Delta_{\mathcal{A}}(\mathcal{X})$, the quotient $\mathcal{X} / \mathcal{P}$ is a one-dimensional Banach left $\mathcal{A}$-module. Denote by $\underline{X}$ the subset in the Cartesian product $\prod_{\mathcal{P} \in \Delta_{\mathcal{A}}(X)} X / \mathcal{P}$ of all $\underline{x}=\left(x_{\mathcal{P}}+\mathcal{P}\right)_{\mathcal{P} \in \Delta_{\mathcal{A}}(X)}$ for which $\|\underline{x}\|=\sup \left\{\left\|x_{\mathcal{P}}+\mathcal{P}\right\| ; \mathcal{P} \in \Delta_{\mathcal{A}}(\mathcal{X})\right\}<\infty$, where the norms in the supremum are the usual quotient norms. It is not hard to see that $\underline{X}$ is a Banach space and it is actually a Banach left $\mathcal{A}$-module for the multiplication given by $a \cdot \underline{x}=\left(a \cdot x_{\mathcal{P}}+\mathcal{P}\right)_{\mathcal{P} \in \Delta_{\mathcal{A}}(x)}$, $a \in \mathcal{A}, \underline{x} \in \underline{X}$.

For each $x \in \mathcal{X}$ and each $\mathcal{P} \in \Delta_{\mathcal{A}}(\mathcal{X})$ denote by $\widehat{x}(\mathcal{P})$ the coset $x+\mathcal{P}$ in $X / \mathcal{P}$. Hence $\widehat{x}$ can be considered as an element in $\underline{X}$. It is clear that $\|\widehat{x}\| \leq\|x\|$ for all $x \in \mathcal{X}$ and that the mapping $\Gamma: x \mapsto \widehat{x}$ is injective if and only if $X$ is hyper-semisimple.

4. Simple multipliers. In this section we shall study a special subclass $M_{\mathcal{A}}(X)$ of multipliers in $B_{\mathcal{A}}(\mathcal{X})$, where $\mathcal{X}$ is a left Banach module over a Banach algebra $\mathcal{A}$. We start with the following proposition (cf. Theorem 1.2.4 in [13]). 
Proposition 4.1. Let $\mathcal{A}$ be a unital Banach algebra and let $\mathcal{X}$ and $Z$ be left Banach $\mathcal{A}$-modules. If $T$ is in $B_{\mathcal{A}}(\mathcal{X}, Z)$, then $T^{*}$ is in $B\left(Z^{*}, X^{*}\right)_{\mathcal{A}}$ and $T^{*}\left(Z_{\varphi}^{\perp}\right) \subseteq X_{\varphi}^{\perp}$ for every $\varphi \in \Sigma(\mathcal{A})$. On the other hand, if $T \in B(X, Z)$ is such that $T^{*}\left(Z_{\varphi}^{\perp}\right) \subseteq X_{\varphi}^{\perp}$ for each $\varphi \in$ $\Sigma(\mathcal{A})$, then $T(a \cdot x)-a \cdot T x$ is in the Gelfand radical of $Z$, for all $a \in \mathcal{A}$ and $x \in \mathcal{X}$. In particular, if $Z$ is hyper-semisimple, then $T \in B_{\mathcal{A}}(X, Z)$.

Proof. The first assertion is obvious. Let $\varphi \in \Sigma(\mathcal{A})$ and $\zeta \in Z_{\varphi}^{\perp}$. Since, by (2), $\left(T^{*} \zeta\right) \cdot a=T^{*}(\zeta \cdot a)=\varphi(a) T^{*} \zeta$ for all $a \in \mathcal{A}$, we have $T^{*} \zeta \in X_{\varphi}^{\perp}$, by Proposition 2.7.

Let $T$ be a bounded linear map from $X$ into $Z$ such that $T^{*}\left(Z_{\varphi}^{*}\right) \subseteq X_{\varphi}^{\perp}$ for all $\varphi \in \Sigma(\mathcal{A})$. Denote by $\mathcal{W}$ the linear span of the union $\cup\left\{\mathcal{Z}_{\varphi}^{\perp} ; \varphi \in \Sigma(\mathcal{A})\right\}$. We have seen (in the proof of Proposition 2.7) that the weak-* closure of $\mathcal{W}$ is $\operatorname{Rad}_{\mathcal{A}}(Z)^{\perp}$. Let $a \in \mathcal{A}$ and $x \in X$ be arbitrary. Then

$$
\begin{aligned}
\langle\xi, T(a \cdot x)-a \cdot T x\rangle & =\sum_{k=1}^{n}\left(\left\langle\xi_{k}, T(a \cdot x)\right\rangle-\left\langle\xi_{k}, a \cdot T x\right\rangle\right) \\
& =\sum_{k=1}^{n}\left(\left\langle\left(T^{*} \xi_{k}\right) \cdot a, x\right\rangle-\left\langle T^{*}\left(\xi_{k} \cdot a\right), x\right\rangle\right) \\
& =\sum_{k=1}^{n}\left(\varphi_{k}(a)\left\langle T^{*} \xi_{k}, x\right\rangle-\varphi_{k}(a)\left\langle T^{*} \xi_{k}, x\right\rangle\right)=0,
\end{aligned}
$$

for $\xi=\sum_{k=1}^{n} \xi_{k} \in \mathcal{W}$, where $\xi_{k} \in X_{\varphi_{k}}^{\perp}, \varphi_{k} \in \Sigma(\mathcal{A}), k=1, \ldots, n$. It follows $T(a \cdot x)-$ $a \cdot T x \in \operatorname{Rad}_{\mathcal{A}}(Z)$, for all $a \in \mathcal{A}$ and $x \in X$.

By the preceding proposition, each $X_{\varphi}^{\perp}, \varphi \in \Sigma(\mathcal{A})$, is invariant for $T^{*}$ if $T \in B_{\mathcal{A}}(\mathcal{X})$. However, since the dimension of $X_{\varphi}^{\perp}$ could be quite large, it is possible that there are too general operators in $B_{\mathcal{A}}(X)$. This is a reason for the following definition.

Definition 4.2. Let $\mathcal{A}$ be a unital Banach algebra and let $\mathcal{X}$ be a left Banach $\mathcal{A}$-module. A multiplier $T$ on $X$ is simple if each closed submodule of co-dimension 1 is invariant for $T$. The set of all simple multipliers will be denoted by $M_{\mathcal{A}}(\mathcal{X})$.

EXAMPLE 4.3. Let $\mathcal{A}$ be a semisimple commutative Banach algebra and let $\mathcal{N}(\mathcal{A}) \subset$ $B(\mathcal{A})$ be the algebra of all multipliers on $\mathcal{A}$. Let us consider $\mathcal{A}$ as a left Banach $\mathcal{A}_{1}$ module. It is obvious that $\Delta_{\mathcal{A}_{1}}(\mathcal{A})$ is exactly the set of all maximal modular ideals in $\mathcal{A}$. Since, by Theorem 1.2.4 in [13], a linear map $T: \mathcal{A} \rightarrow \mathcal{A}$ is in $\mathcal{M}(\mathcal{A})$ if and only if $T M \subseteq M$, for every maximal modular ideal $M$ in $\mathcal{A}$, we conclude that $\mathcal{N}(\mathcal{A})=$ $M_{\mathcal{A}_{1}}(\mathcal{A})$; i.e., all multipliers on $\mathcal{A}$ are simple.

The preceding example shows that sometimes all multipliers are simple. It is obvious that this is the case if each set $\Delta_{\varphi}(X), \varphi \in \Sigma(\mathcal{A})$, is either empty or a singleton. In the next example we shall see that sometimes the class of simple multipliers coincides with a class of operators which is in general much bigger.

EXAMPLE 4.4. Let $\mathcal{A}$ be a unital commutative Banach algebra and $X$ a left Banach $\mathcal{A}$-module. Denote by $\vartheta$ the corresponding representation of $\mathcal{A}$ on $\mathcal{X}$, i.e. $\vartheta(a) x=a \cdot x$ for all $a \in \mathcal{A}, x \in \mathcal{X}$. The local Arveson spectrum of $\vartheta$ at $x \in \mathcal{X}$ is defined as

$$
\operatorname{sp}_{\vartheta}(x):=\left\{\varphi \in \Sigma(\mathcal{A}) ; \varphi(a)=0 \quad \text { for all } a \in \operatorname{ann}_{\mathcal{A}}(x)\right\},
$$


(see $\S 4.12$ in [18]). It is easily seen that $s p_{\vartheta}(x)$ is empty if and only if $x=0$. If $\mathcal{A}$ is regular (in the sense of Shilov) and semisimple and $\lambda$ is the left regular representation of $\mathcal{A}$, then, for each $a \in \mathcal{A}$, the local Arveson spectrum $\operatorname{sp}_{\lambda}(a)$ coincides with the support supp $\widehat{a}$ of the Gelfand transform of $a$.

We shall say that a bounded linear operator $T$ on $X$ is local if $s p_{\vartheta}(T x) \subseteq s p_{\vartheta}(x)$ holds for all $x \in \mathcal{X}$ (cf. [10] and [11, Lemma 1]). It is not hard to see that each multiplier is a local operator. On the other hand, there exist local operators which are not multipliers (for instance, if $\Sigma(\mathcal{A})$ is a singleton, then every bounded operator is local but not necessarily a multiplier).

We confine ourselves to the case $\mathcal{A}=\mathcal{C}(K), \mathcal{X}=\mathcal{A}^{*}$, where $K$ is a compact Hausdorff space. Thus, $X$ is the dual module of $\mathcal{A}$ and its elements can be considered, by the Riesz representation theorem, as finite regular Borel measures on $K$. It is well known that $\Sigma(\mathcal{A})=K$; the character that corresponds to $t \in K$ is the point mass at $t$.

It follows from Theorem 5 in [25] that every local operator on $X$ is a multiplier. Now we shall show that, for $t \in K$, each set $\Delta_{t}(X)$ is a singleton, which enables us to conclude that all multipliers on $X$ are simple.

Since $\mathcal{A}$ is amenable [5, Theorem 5.6.2 (i)] there exists a non-trivial point multiplier on $X$ at every $t \in K$, by [1, Proposition $4 \S 43]$. Thus, $\Delta_{t}(X)(t \in K)$ are not empty. On the other hand, assume that $F, G \in X^{*}=\mathcal{A}^{* *}$ are two non-trivial point multipliers at $t \in K$. Since the algebra $\mathcal{A}$ is Arens regular (see [5]) there is only one Arens product on $\mathcal{A}^{* *}$ and it is commutative. Let $F \cdot G$ denote the Arens product of $F$ and $G$. Using formulae (2.6.27) in [5] it is straightforward to see that

$$
\left\langle F, \delta_{t}\right\rangle G=F \cdot G=\left\langle G, \delta_{t}\right\rangle F,
$$

where $\delta_{t}$ is the point mass at $t$. Thus, $\Delta_{t}(X)$ is indeed a singleton.

Now we proceed with the general theory of simple multipliers. Since the proof of the next proposition is almost obvious we shall omit it.

Proposition 4.5. Let $\mathcal{A}$ be a unital Banach algebra and let $X$ be a Banach left $\mathcal{A}$-module. The set $M_{\mathcal{A}}(X)$ is a subalgebra in $B_{\mathcal{A}}(X)$. It is closed in the strong operator topology, and it contains any multiplication operator induced by an element from the center of $\mathcal{A}$, in particular, the identity operator $I$ is always in $M_{\mathcal{A}}(X)$.

It is obvious that a multiplier $T \in B_{\mathcal{A}}(X)$ is simple if and only if for each $\varphi \in \Sigma(\mathcal{A})$ and each $\xi \in X_{\varphi}^{\perp}$, there exists a number $\lambda_{\xi} \in \mathbb{C}$ such that $T^{*} \xi=\lambda_{\xi} \xi$. If $\xi$ and $\eta$ are arbitrary non-zero point multipliers at $\varphi$, then $\lambda_{\alpha \xi+\beta \eta}(\alpha \xi+\beta \eta)=T^{*}(\alpha \xi+\beta \eta)=$ $\alpha \lambda_{\xi} \xi+\beta \lambda_{\eta} \eta$, for all $\alpha, \beta \in \mathbb{C}$, shows that $\lambda_{\xi}$ does not depend on $\xi$ but on $\varphi$, i.e., there is $\lambda_{\varphi} \in \mathbb{C}$ such that $T^{*} \xi=\lambda_{\varphi} \xi$ for all $\xi \in X_{\varphi}^{\perp}$. Define, for $T \in M_{\mathcal{A}}(X)$, a complex function $\widetilde{T}$ on $\Delta_{\mathcal{A}}(\mathcal{X})$ by $\widetilde{T}(\mathcal{P})=\lambda_{\nu_{\mathcal{A}}(\mathcal{P})}, \mathcal{P} \in \Delta_{\mathcal{A}}(\mathcal{X})$. It is not hard to see that $\widetilde{T}(\mathcal{P})=$ $\langle\xi, x\rangle^{-1}\langle\xi, T x\rangle$ if $\xi$ is a non-zero point multiplier in $\mathcal{P}^{\perp}$ and $x \in \mathcal{X}$ is such that $\langle\xi, x\rangle \neq 0$. Note that $\widetilde{T}$ is constant on each set $\Delta_{\varphi}(\mathcal{X}), \varphi \in \Sigma(\mathcal{A})$, and that in the special case, when $T$ is a multiplication by $a \in \mathcal{A}$, we have $\widetilde{T}(\mathcal{P})=\widehat{a}\left(v_{\mathcal{A}}(\mathcal{P})\right)$.

Let us define $\|\widetilde{T}\|_{\infty}:=\sup \left\{|\widetilde{T}(\mathcal{P})| ; \mathcal{P} \in \Delta_{\mathcal{A}}(\mathcal{X})\right\}$ for $T \in M_{\mathcal{A}}(\mathcal{X})$. Then $\|\widetilde{T}\|_{\infty}$ is a finite number. Moreover, following the proof of Theorem 1.2.2 in [13], we can prove the estimate $\|\widetilde{T}\|_{\infty} \leq\|T\|$. The next proposition can be proven in a similar way as Theorem 1.2.2 (i) and Theorem 1.1.3 in [13].

Proposition 4.6. Let $\mathcal{A}$ be a unital Banach algebra, $X$ a Banach left $\mathcal{A}$-module, and let $T \in M_{\mathcal{A}}(\mathcal{X})$. 
(a) For all $x \in X$ and all $\mathcal{P} \in \Delta_{\mathcal{A}}(\mathcal{X})$ we have $(T x) \Upsilon(\mathcal{P})=\widetilde{T}(\mathcal{P}) \widehat{x}(\mathcal{P})$.

(b) If $T$ is bijective, then $T^{-1}$ is in $M_{\mathcal{A}}(\mathcal{X})$ and $T^{-1}(\mathcal{P})=\widetilde{T}(\mathcal{P})^{-1}$ for all $\mathcal{P} \in \Delta_{\mathcal{A}}(\mathcal{X})$.

ExAmple 4.7. Let $\mathcal{X}$ be a complex Banach space and $A \in B(\mathcal{X})$. Denote by $\mathcal{A}$ the closed subalgebra in $B(X)$ which is generated by $A$ and the identity operator, i.e., the closure in $B(X)$ of the set

$\{p(A) ; p$ is a complex polynomial $\}$.

Of course, $\mathcal{A}$ is a unital commutative Banach algebra. It is well known that $\Sigma(\mathcal{A})$ can be identified by the set $\widehat{\sigma(A)}$, the polynomially convex hull of the spectrum of $A$ (see, for instance, Proposition VII.8.10 and Theorem VII.5.4 (d) in [4]). In particular, the Gelfand transform of $p(A) \in \mathcal{A}$ is given by $\widehat{p(A)}(\lambda)=p(\lambda), \lambda \in \widehat{\sigma(A)}$.

Consider $X$ as a left Banach $\mathcal{A}$-module via the multiplication given by $S \cdot x=S x$, $S \in \mathcal{A}, x \in \mathcal{X}$. If $\xi \in X^{*}$ is a nontrivial point multiplier at $\lambda \in \widehat{\sigma(A)}$, then

$$
\langle\xi, A x\rangle=\widehat{A}(\lambda)\langle\xi, x\rangle=\lambda\langle\xi, x\rangle \quad(x \in X),
$$

which implies $A^{*} \xi=\lambda \xi$. Hence, $\lambda$ is an eigenvalue of $A^{*}$ and $\xi$ is an eigenvector at $\lambda$. On the other hand, it is a routine to show that if $\lambda$ is an eigenvalue of $A^{*}$ and $0 \neq \xi \in X^{*}$ is in the null space of $A^{*}-\lambda$, then $\xi$ is a point multiplier at $\lambda$. Thus, we may conclude that the image of $\Delta_{\mathcal{A}}(\mathcal{X})$ under the natural map $\nu_{\mathcal{A}}$ is $\sigma_{p}\left(A^{*}\right)$, the point spectrum of $A^{*}$.

For a simple multiplier $T \in M_{\mathcal{A}}(\mathcal{X})$, let $t: \sigma_{p}\left(A^{*}\right) \rightarrow \mathbb{C}$ be the function defined by $t(\lambda):=\widetilde{T}(\mathcal{P})\left(\lambda \in \sigma_{p}\left(A^{*}\right)\right)$ where $\mathcal{P}$ is an arbitrary submodule in $\Delta_{\lambda}(\mathcal{X})$. Since $T^{*} \xi=$ $t(\lambda) \xi$, for any point multiplier $\xi$ at $\lambda \in \sigma_{p}\left(A^{*}\right)$, we have inclusions

$$
\operatorname{ker}\left(A^{*}-\lambda\right) \subseteq \operatorname{ker}\left(T^{*}-t(\lambda)\right) \quad\left(\lambda \in \sigma_{p}\left(A^{*}\right)\right) .
$$

On the other hand, it is almost obvious that $T \in B_{\mathcal{A}}(X)$ is a simple multiplier if, for each $\lambda \in \sigma_{p}\left(A^{*}\right)$, there exists a number $t(\lambda) \in \mathbb{C}$ such that (4) is fulfilled.

Let $1 \leq p<\infty$ and let $q>1$ be such that $1 / p+1 / q=1$ (we set $q=\infty$ when $p=1$ ). Denote by $A: \ell^{p} \rightarrow \ell^{p}$ the unilateral shift. It is well known (see $[4, \S$ VII.6]) that $\sigma(A)=\overline{\mathbb{D}}$, the closed unit disc in $\mathbb{C}$. The adjoint of $A$ is the backward shift $A^{*}: \ell^{q} \rightarrow \ell^{q}$. The point spectrum of $A^{*}$ contains $\mathbb{D}$ and, for each $\lambda \in \mathbb{D}$, the null space $\operatorname{ker}\left(A^{*}-\lambda\right)$ is the one-dimensional space spanned by the vector $\left(1, \lambda, \lambda^{2}, \ldots\right)$. Now it is not hard to see that $\ell^{p}$ is a hyper-semisimple left Banach $\mathcal{A}$-module, where $\mathcal{A}$ is the closed subalgebra in $B\left(\ell^{p}\right)$ generated by $A$ and the identity operator. Note that $B_{\mathcal{A}}\left(\ell^{p}\right)=M_{\mathcal{A}}\left(\ell^{p}\right)$ because of $\operatorname{dim}\left(\operatorname{ker}\left(A^{*}-\lambda\right)\right)=1\left(\lambda \in \sigma_{p}\left(A^{*}\right)\right)$.

Consider a left Banach $\mathcal{A}$-module $\mathcal{X}$ also as a left Banach $M_{\mathcal{A}}(\mathcal{X})$-module the multiplication is defined by $T \cdot x:=T x, T \in M_{\mathcal{A}}(X), x \in \mathcal{X}$. In the dual Banach $M_{\mathcal{A}}(X)$-module $X^{*}$ we have $\xi \cdot T=\widetilde{T}(\mathcal{P}) \xi$, for all $\xi \in \mathcal{P}^{\perp}, \mathcal{P} \in \Delta_{\mathcal{A}}(\mathcal{X})$. By definition, all $\mathcal{P} \in \Delta_{\mathcal{A}}(\mathcal{X})$ are $T$-invariant for each $T \in M_{\mathcal{A}}(\mathcal{X})$, hence $\Delta_{\mathcal{A}}(X) \subseteq \Delta_{M_{\mathcal{A}}(X)}(\mathcal{X})$. The next example shows that this inclusion can be proper.

EXAMPLE 4.8. Let $X$ be a Banach space of dimension greater than 1 and let $\mathcal{A}=B(X)$. The space $X$ is a left Banach $\mathcal{A}$-module in the usual way. It is obvious that $\Delta_{\mathcal{A}}(X)$ is empty. Of course, in $B_{\mathcal{A}}(\mathcal{X})$, and therefore in $M_{\mathcal{A}}(X)$, are only the scalar multiples of the identity operator. Hence $\Delta_{M_{\mathcal{A}}(X)}(X)$ consists of all subspaces in $\mathcal{X}$ of co-dimension 1. 
Since $X$ is simultaneously considered as a left Banach $\mathcal{A}$ - and $M_{\mathcal{A}}(X)$-module, we will denote hulls and kernels by $H_{X}$ and $K_{X}$ when $X$ is $M_{\mathcal{A}}(X)$-module.

Proposition 4.9. Let $\mathcal{A}$ be a unital Banach algebra and let $X$ be a Banach left $\mathcal{A}$-module as well as a left Banach $M_{\mathcal{A}}(\mathcal{X})$-module. Then $\Delta_{\mathcal{A}}(X) \subseteq \Delta_{M_{\mathcal{A}}}(X)(X)$ and the hull-kernel topology on $\Delta_{\mathcal{A}}(X)$ is the relative hull-kernel topology, which $\Delta_{\mathcal{A}}(X)$ inherits from $\Delta_{M_{\mathcal{A}}(X)}(X)$, that is $S=H_{X}\left(K_{X}(S)\right) \cap \Delta_{\mathcal{A}}(X)$ for each hull-kernel closed subset $S \subseteq$ $\Delta_{\mathcal{A}}(X)$. If $\mathcal{A}$ is commutative, then $\Delta_{\mathcal{A}}(X)=\Delta_{M_{\mathcal{A}}}(X)(X)$ and both hull-kernel topologies coincide.

Proof. It is obvious that $S \subseteq H_{X}\left(K_{X}(S)\right) \cap \Delta_{\mathcal{A}}(X)$. On the other hand, if $\mathcal{P} \in$ $H_{X}\left(K_{X}(S)\right) \cap \Delta_{\mathcal{A}}(X)$, then $\left(K_{X}(S): X\right) \subseteq(\mathcal{P}: \mathcal{X})$. However, $K_{X}(S)=k_{X}(S)$ and hence $\mathcal{P} \in h_{X}\left(k_{X}(S)\right)=S$.

If $\mathcal{A}$ is commutative, then every multiplication operator $T_{a}$ on $\mathcal{X}, a \in \mathcal{A}$, is in $M_{\mathcal{A}}(X)$. Thus, if $\mathcal{P}$ is in $\Delta_{M_{\mathcal{A}}(X)}(\mathcal{X})$, it is also in $\Delta_{\mathcal{A}}(X)$.

Corollary 4.10. Let $\mathcal{A}$ be a unital Banach algebra and let $X$ be a Banach left $\mathcal{A}$-module. If $\mathcal{X}$ is hyper-semisimple, then $M_{\mathcal{A}}(X)$ is a semisimple commutative Banach algebra.

Proof. Let $S$ and $T$ be in $M_{\mathcal{A}}(X)$ and let $x$ be an arbitrary vector in $X$. Since $\mathcal{X}$ is hyper-semisimple it follows from $(S T x)(\mathcal{P})=\widetilde{S}(\mathcal{P}) \widetilde{T}(\mathcal{P}) \widehat{x}(\mathcal{P})=(T S x)(\mathcal{P})$, $\mathcal{P} \in \Delta_{\mathcal{A}}(\mathcal{X})$, that $S T x=T S x$. Thus, $M_{\mathcal{A}}(\mathcal{X})$ is a unital commutative Banach algebra. The radical $\operatorname{Rad}_{M_{\mathcal{A}}(X)}\left(M_{\mathcal{A}}(X)\right)$ coincides with the ordinary Gelfand radical of $M_{\mathcal{A}}(X)$, because $M_{\mathcal{A}}(X)$ is commutative and, by Example 2.2, in $\Delta_{M_{\mathcal{A}}(X)}\left(M_{\mathcal{A}}(X)\right)$ are exactly the maximal ideals $M_{\varphi}, \varphi \in \Sigma\left(M_{\mathcal{A}}(\mathcal{X})\right)$. Consider $\mathcal{X}$ as a Banach left $M_{\mathcal{A}}(\mathcal{X})$-module and assume that $T$ is in $\operatorname{Rad}_{M_{\mathcal{A}}(X)}\left(M_{\mathcal{A}}(\mathcal{X})\right)$. Then $T X \subseteq \mathcal{P}$ for each $\mathcal{P} \in \Delta_{M_{\mathcal{A}}(X)}(X)$ because $\langle\xi, T x\rangle=\varphi(T)\langle\xi, x\rangle=0, \varphi \in \Sigma\left(M_{\mathcal{A}}(\mathcal{X})\right), \xi \in C h_{\varphi}\left(\mathcal{X}^{*}\right)$. Thus, by hyper-semisimplicity of $\mathcal{X}$, the subspace $T \mathcal{X}$ is trivial and hence $T=0$.

When $X$ is considered as a left Banach $\mathcal{A}$ - and $M_{\mathcal{A}}(\mathcal{X})$-module, then, for $T \in$ $M_{\mathcal{A}}(X)$, there are defined two functions $\widetilde{T}: \Delta_{\mathcal{A}}(X) \rightarrow \mathbb{C}$ and, say, $\widetilde{T}: \Delta_{M_{\mathcal{A}}(X)}(X) \rightarrow \mathbb{C}$. However, it is easily seen that $\widetilde{T}$ is just the restriction of $\widetilde{\widetilde{T}}$ to $\Delta_{\mathcal{A}}(X)$. From now on we shall denote by $\widetilde{T}$ both $\widetilde{\widetilde{T}}$ and its restriction to $\Delta_{\mathcal{A}}(X)$.

Let $v_{M}$ be the natural map from $\Delta_{M_{\mathcal{A}}(X)}(\mathcal{X})$ into $\Sigma\left(M_{\mathcal{A}}(\mathcal{X})\right)$. If $T$ is a simple multiplier and $\widehat{T}: \Sigma\left(M_{\mathcal{A}}(X)\right) \rightarrow \mathbb{C}$ is its Gelfand transform, then $\widetilde{T}=\widehat{T} \circ v_{M}$. Thus, by Proposition 3.5, $\widetilde{T}$ is hull-kernel continuous if and only if $\widehat{T}$ is continuous with respect to the relative hull-kernel topology on $v_{M}\left(\Delta_{M_{\mathcal{A}}(X)}(X)\right)$.

5. Spectral properties of simple multipliers. Recall some basic notions from the local spectral theory of operators. The reader is referred to the excellent monograph [18] for more details.

A bounded linear operator $T$ on a Banach space $X$ has the single-valued extension property (SVEP) if for every open $U \subseteq \mathbb{C}$ the only analytic solution $f: U \rightarrow X$ of the equation $(\lambda-T) f(\lambda)=0(\lambda \in U)$ is $f \equiv 0$.

An operator $T \in B(X)$ is said to have the decomposition property $(\delta)$ if, given an arbitrary open covering $\left\{U_{1}, U_{2}\right\}$ of $\mathbb{C}$, every $x \in \mathcal{X}$ admits a decomposition $x=$ $u_{1}+u_{2}$ where the vector $u_{k}(k=1,2)$ satisfies $u_{k}=(T-\lambda) f_{k}(\lambda)$ for all $\lambda \in \mathbb{C} \backslash \bar{U}_{k}$ and some analytic function $f_{k}: \mathbb{C} \backslash \bar{U}_{k} \rightarrow X$. 
If for every open covering $\{U, V\}$ of $\mathbb{C}$ there exists a pair of closed linear subspaces $y$ and $z$ in $X$ such that they are invariant for $T \in B(X)$ and $X=y+z, \sigma(T \mid y) \subseteq U$, $\sigma\left(\left.T\right|_{z}\right) \subseteq V$, then $T$ is called decomposable (cf. [3]). When the sum $y+z$ is just dense in $X$, then $T$ has the weak 2-spectral decomposability property (weak 2-SDP). A stronger notion than decomposability is super-decomposability. An operator $T \in B(X)$ is superdecomposable if for every open covering $\{U, V\}$ of $\mathbb{C}$ there exists some $S \in B(X)$, commuting with $T$, such that $\sigma\left(T_{\overline{i m S}}\right) \subseteq U$ and $\sigma\left(T_{\overline{i m(I-S)}}\right) \subseteq V$, (see [15]).

By a simple modification of the proof of Proposition 6.2.3 in [3] we get the following assertion.

Proposition 5.1. Let $\mathcal{A}$ be a unital Banach algebra and let $\mathcal{X}$ be a hyper-semisimple Banach left $\mathcal{A}$-module. Then every $T \in M_{\mathcal{A}}(X)$ has the single-valued extension property.

An immediate consequence is the following well known assertion.

COROLlary 5.2. If $T \in B\left(\ell^{p}\right)(1 \leq p<\infty)$ commutes with the unilateral shift, then it has single-valued extension property.

Proof. See the end of Example 4.7 and use the previous proposition.

The next theorem extends a part of Proposition 3 in [16] and a part of Proposition 1 in $[\mathbf{8}]$ to Banach modules. The proof is a modification of the proofs in $[\mathbf{1 6}, \mathbf{8}]$. However, since the concept of point multipliers is used, we give the details.

Theorem 5.3. Let $\mathcal{A}$ be a unital Banach algebra and let $X$ be a left Banach $\mathcal{A}$ module. If $T \in M_{\mathcal{A}}(X)$ has the decomposition property $(\delta)$ or the weak 2-SDP, then $\widehat{T}: \Sigma\left(M_{\mathcal{A}}(X)\right) \rightarrow \mathbb{C}$ is continuous on $v_{M}\left(\Delta_{M_{\mathcal{A}}(X)}(X)\right)$ with respect to the relative hullkernel topology.

Proof. Assume that $\widehat{T}$ is not continuous on $v_{M}\left(\Delta_{M_{\mathcal{A}}(\mathcal{X})}(\mathcal{X})\right)$ with respect to the relative hull-kernel topology. Then $\widetilde{T}$ is not continuous with respect to the hullkernel topology on $\Delta_{M_{\mathcal{A}}(X)}(\mathcal{X})$. Hence there exists a closed subset $F$ of $\mathbb{C}$ such that $\mathcal{F}:=\left\{\mathcal{P} \in \Delta_{M_{\mathcal{A}}(X)}(\mathcal{X}) ; \widetilde{T}(\mathcal{P}) \in F\right\}$ is not hull-kernel closed in $\Delta_{M_{\mathcal{A}}(X)}(\mathcal{X})$. Choose $\mathcal{P}_{0} \in$ $H_{x}\left(K_{x}(\mathcal{F})\right) \backslash \mathcal{F}$ and denote $\lambda_{0}=\widetilde{T}\left(\mathcal{P}_{0}\right) \notin F$. Let $V_{1}$ and $V_{2}$ be open neighbourhoods of $\lambda_{0}$, respectively of $F$, such that $\bar{V}_{1} \cap \bar{V}_{2}=\emptyset$. Denote $U_{k}=\left(\bar{V}_{k}\right)^{c}, k=1,2$. Then $\left\{U_{1}, U_{2}\right\}$ is an open covering of $\mathbb{C}$.

Consider first the case when $T$ has the decomposition property $(\delta)$. A given $x \in \mathcal{X}$ can be written as $x=u_{1}+u_{2}$, where $u_{k}=(T-\lambda) f_{k}(\lambda)$ for all $\lambda \in \mathbb{C} \backslash \bar{U}_{k}$ and some analytic function $f_{k}: \mathbb{C} \backslash \bar{U}_{k} \rightarrow X, k=1,2$. The point $\lambda_{0}$ is in $\mathbb{C} \backslash \bar{U}_{1}$ therefore we have $u_{1}=\left(T-\lambda_{0}\right) f_{1}\left(\lambda_{0}\right)$. Denote $\varphi_{0}=v_{M}\left(\mathcal{P}_{0}\right) \in \Sigma\left(M_{\mathcal{A}}(X)\right)$ and let $\xi$ be an arbitrary point multiplier in $\mathcal{P} \perp$. Then $\left\langle\xi, u_{1}\right\rangle=\left\langle\xi,\left(T-\lambda_{0}\right) f_{1}\left(\lambda_{0}\right)\right\rangle=\varphi_{0}\left(T-\lambda_{0}\right)\left\langle\xi, f_{1}\left(\lambda_{0}\right)\right\rangle=0$ and hence $u_{1} \in \mathcal{P}_{0}$.

Now let $\mathcal{P}$ be an arbitrary point in $\mathcal{F}$. Since $\mu:=\widetilde{T}(\mathcal{P}) \in F$, we have $u_{2}=(T-$ u) $f_{2}(\mu)$. The multiplier $T-\mu$ is in $k_{M_{\mathcal{A}}(X)}\left(\left\{v_{M}(\mathcal{P})\right\}\right)$, therefore $u_{2} \in k_{M_{\mathcal{A}}(X)}\left(\left\{v_{M}(\mathcal{P})\right\}\right)$. $x$. It follows

$$
\begin{aligned}
u_{2} \in \bigcap_{\mathcal{P} \in \mathcal{F}}\left(k_{M_{\mathcal{A}}(X)}\left(\left\{v_{M}(\mathcal{P})\right\}\right) \cdot X\right) & =\left(\bigcap_{\mathcal{P} \in \mathcal{F}} k_{M_{\mathcal{A}}(X)}\left(\left\{v_{M}(\mathcal{P})\right\}\right)\right) \cdot X \\
& =k_{M_{\mathcal{A}}(X)}\left(v_{M}(\mathcal{F})\right) \cdot X
\end{aligned}
$$


Since $\mathcal{P}_{0}$ is in $H_{X}\left(K_{X}(\mathcal{F})\right)$ and, by Proposition 3.4 (ii), $k_{M_{\mathcal{A}}(X)}\left(v_{M}(\mathcal{F})\right)=\left(K_{X}(\mathcal{F}): X\right)$, the vector $u_{2}$ must be in $k_{M_{\mathcal{A}}(X)}\left(v_{M}(\mathcal{F})\right) \cdot X \subseteq \mathcal{P}_{0}$. Thus, $x=u_{1}+u_{2} \in \mathcal{P}_{0}$ for all $x \in \mathcal{X}$ a contradiction.

Assume that $T$ has the weak 2-SDP. Then there exist $T$-invariant subspaces $y$ and $Z$ such that $\sigma\left(\left.T\right|_{y}\right) \subseteq U_{1}, \sigma\left(\left.T\right|_{z}\right) \subseteq U_{2}$ and $\overline{y+z}=X$. An arbitrary $y \in \mathcal{y}$ can be written as $y=\left(\left.T\right|_{y}-\lambda_{0}\right) u=\left(T-\lambda_{0}\right) u$ for some $u \in y$ since $\lambda_{0} \notin U_{1}$. If $\xi$ is in $\mathcal{P}_{0}^{\perp}$, then $\left\langle\xi, u_{2}\right\rangle=\left\langle\xi,\left(T-\lambda_{0}\right) u\right\rangle=0$, that is $y \in \mathcal{P}_{0}$. For an arbitrary $\mathcal{P} \in \mathcal{F}$ let $\mu:=$ $\widetilde{T}(\mathcal{P}) \in F$. Each $z \in Z$ is of the form $z=\left(\left.T\right|_{z}-\mu\right) v=(T-\mu) v$ for some $v \in Z$, that is $z \in k_{M_{\mathcal{A}}(X)}\left(\left\{v_{M}(\mathcal{P})\right\}\right) \cdot \mathcal{X}$, because of $\mu \notin U_{2}$. It follows $z \in k_{\mathcal{A}}\left(v_{M}(\mathcal{F})\right) \cdot X \subseteq \mathcal{P}_{0}$ as before. Thus, $y+z \subseteq \mathcal{P}_{0}$ and we have a contradiction.

We shall say about a simple multiplier $T$ that it has natural spectrum when $\sigma(T)=$ $\widetilde{T}\left(\Delta_{\mathcal{A}}(X)\right)$. The next theorem extends the remained parts of Proposition 3 in [16] and Proposition 1 in $[8]$ to Banach modules.

THEOREM 5.4. Let $\mathcal{A}$ be a unital Banach algebra and let $\mathcal{X}$ be a hyper-semisimple left Banach $\mathcal{A}$-module. If $T \in M_{\mathcal{A}}(X)$ has the decomposition property $(\delta)$ or weak 2-SDP, then it has natural spectrum.

Proof. It is easy to see that $\left(x_{\mathcal{P}}+\mathcal{P}\right)_{\mathcal{P} \in \Delta_{\mathcal{A}}(X)} \mapsto\left(\widetilde{T}(\mathcal{P}) x_{\mathcal{P}}+\mathcal{P}\right)_{\mathcal{P} \in \Delta_{\mathcal{A}}(X)}$ defines a bounded linear operator $S$ on $\underline{x}$. It is also not hard to show that $\Gamma T=S \Gamma$, where $\Gamma$ is the map that is defined at the end of Section 3 . Since $X$ is hyper-semisimple the multiplier $T$ has SVEP and $\Gamma$ is injective, as we remarked at the end of Section 3. Thus, if $T$ has $(\delta)$, we can use Lemma 1 in [16], which asserts that $\sigma(T) \subseteq \sigma(S)$. The same is true if $T$ has weak 2-SDP, by Lemma 1 in [7]. On the other hand, it is almost evident that $\sigma(S) \subseteq \overline{\widetilde{T}\left(\Delta_{\mathcal{A}}(\mathcal{X})\right)} \subseteq \sigma(T)$. Indeed, if $\lambda$ is not in $\widetilde{T}\left(\Delta_{\mathcal{A}}(\mathcal{X})\right)$, then $S_{\lambda}:\left(x_{\mathcal{P}}+\mathcal{P}\right)_{\mathcal{P} \in \Delta_{\mathcal{A}}(X)} \mapsto\left((\widetilde{T}(\mathcal{P})-\lambda)^{-1} x_{\mathcal{P}}+\mathcal{P}\right)_{\mathcal{P} \in \Delta_{\mathcal{A}}(X)}$ is a bounded linear operator on $\underline{X}$ such that $S_{\lambda}(S-\lambda)=(S-\lambda) S_{\lambda}=I$. This proves the first inclusion. The second inclusion follows from the fact that $(\widetilde{T}-\lambda)^{-1}$ cannot exist if $\lambda$ is in $\widetilde{T}\left(\Delta_{\mathcal{A}}(\mathcal{X})\right.$ ) (see Proposition 4.6 (b)).

The last result is an extension of a well-known result of M. M. Neumann, see [23] or [22].

THEOREM 5.5. Let $\mathcal{A}$ be a unital Banach algebra and let $\mathcal{X}$ be a hyper-semisimple left Banach $\mathcal{A}$-module. If $T \in M_{\mathcal{A}}(X)$ is such that its Gelfand transform $\widehat{T}: \Sigma\left(M_{\mathcal{A}}(X)\right) \rightarrow \mathbb{C}$ is hull-kernel continuous, then $T$ is super-decomposable.

Proof. Since $X$ is a hyper-semisimple Banach left $\mathcal{A}$-module, it follows, by Corollary 4.10 , that $M_{\mathcal{A}}(X)$ is a semisimple commutative Banach algebra. Hence, by Theorem 1.2 of [23], the multiplication operator $L_{T}: M_{\mathcal{A}}(X) \rightarrow M_{\mathcal{A}}(X)$ is super-decomposable. Since $M_{\mathcal{A}}(X)$ is a closed subalgebra in $B(X)$, Theorem 3.2 of [15], which asserts that $T$ is super-decomposable, can be used.

Acknowledgement. The author would like to thank Professor Milan Hladnik and Professor Roman Drnovšek for stimulating conversations and for a careful reading of the first version of this paper. 


\section{REFERENCES}

1. F. F. Bonsal and J. Duncan, Complete normed algebras (Springer-Verlag, 1973).

2. J. Bračič, Representations and derivations of modules, Irish Math. Soc. Bulletin 47 (2001), 27-39.

3. I. Colojoară and C. Foiaş, Theory of generalized spectral operators (Gordon and Breach, New York, 1968).

4. J. B. Conway, A course in functional analysis (Springer-Verlag, 1985).

5. H. G. Dales, Banach algebras and automatic continuity (Clarendon Press, Oxford, 2000).

6. R. S. Doran and J. Wichmann, Approximate identities and factorization in Banach modules (Springer-Verlag, 1979).

7. J. Eschmeier, Operator decomposability and weakly continuous representations of locally compact abelian groups, J. Operator Theory 7 (1982), 201-208.

8. J. Eschmeier, K. B. Laursen and M. M. Neumann, Multipliers with natural local spectra on commutative Banach algebra, J. Funct. Anal. 138 (1996), 273-294.

9. Şt. Frunză, A characterization of regular Banach algebras, Rev. Roum. Math. Pures Appl. 18 (1973), 1057-1059.

10. B. E. Johnson, Local derivations on $C^{*}$-algebras are derivations, Trans. Amer. Math. Soc., 353 (2000), 313-325.

11. R. Kantrowitz and M. M. Neumann, Disjointness preserving and local operators on algebras of differentiable functions, Glasgow Math. J. 43 (2001), 295-309.

12. J. W. Kitchen and D. A. Robbins, Gelfand representation of Banach modules, Dissertationes Math. (Rozprawy matematyczne) 203 (1982).

13. R. Larsen, An Introduction to the theory of multipliers (Springer-Verlag, 1971).

14. K. B. Laursen, Multipliers and local spectral theory, Functional analysis and operator theory, Banach Center Publications 30 (1994), 223-236.

15. K. B. Laursen and M. M. Neumann, Decomposable operators and automatic continuity, J. Operator Theory 15 (1986), 33-51.

16. K. B. Laursen and M. M. Neumann, Local spectral properties of multipliers on Banach algebras, Arch. Math. (Basel) 58 (1992), 368-375.

17. K. B. Laursen and M. M. Neumann, Decomposable multipliers and applications to harmonic analysis, Studia Math. 101 (1992), 193-214.

18. K. B. Laursen and M. M. Neumann, An introduction to local spectral theory (Clarendon Press, Oxford, 2000).

19. C.-P. Lu, Spectra of modules, Comm. Algebra 23 (1995), 3741-3752.

20. C.-P. Lu, The Zariski topology on the prime spectrum of a module, Houston J. Math. 25 (1999), 417-432.

21. R. L. McCasland, M. E. Moore and P. F. Smith, On the spectrum of a module over a commutative ring, Comm. Algebra 25 (1997), 79-103.

22. M. M. Neumann, Banach algebras, decomposable convolution operators, and a spectral mapping property, in Proceedings of the Conference of Function Spaces at Southern Illinois University at Edwardsville (Marcel Dekker, New York, 1991), 307-323.

23. M. M. Neumann, Commutative Banach algebras and decomposable operators, Monath. Math. 113 (1992), 227-243.

24. T. W. Palmer, Banach algebras and the general theory of $*$-algebras, Volume 1: Algebras and Banach algebras (Cambridge University Press, 1994).

25. V. S. Shulman, spectral synthesis and the Fuglede-Putnam-Rosenblum theorem, Teor. Funkts., Funkts. Anal. Prilozh 54 (1990), 25-36 (in Russian).

26. P. F. Smith, Primary modules over commutative rings, Glasgow Math. J. 43 (2001), $103-111$. 